\title{
The search for a link between autism and neurodegenerative conditions
}

\author{
BY GIORGIA GUGLIELMI
}

28 SEPTEMBER 2021

\section{Listen to this story:}

https://www.spectrumnews.org/wp-content/uploads/2021/09/audio-5165c78beeb0-4c63-ac96-a8eeb6fe755d-encodings.mp3

About five years ago, when his younger twin brothers reached their thirties, Giacomo Vivanti started to wonder how the pair, who both have autism, would fare in middle and old age. In particular, he wondered if they might be prone to develop age-related neurological conditions.

His brothers didn't show any signs of ill health or cognitive deterioration, but Vivanti, associate professor of early detection and intervention at the A.J. Drexel Autism Institute in Philadelphia, Pennsylvania, knew that the scientific literature provided few clear answers.

"I was pretty shocked to learn that we have such limited knowledge of outcomes as children with autism become adults, and as they age," Vivanti says.

It prompted him to scour four years' worth of data from Medicaid, the largest healthcare program in the United States, to determine the incidence of neurodegenerative conditions among 30- to 64-year-olds with autism. That group, he and his colleagues reported last month, is about 2.5 times as likely to be diagnosed with early-onset Alzheimer's or other forms of dementia as the general population.

The study is one of a handful that have found higher-than-average rates of neurodegenerative conditions in autistic adults. The risk estimates for Parkinson's disease in autistic people range from 15 to 20 percent, compared with about 1 percent in the general population. Similarly, the prevalence of dementia is less than 1 percent in non-autistic people but about 4 percent in those with autism. None of these studies offer solid evidence, but their results are strong enough to warrant further investigation, researchers say. 


\section{Spectrum | Autism Research News}

https://www.spectrumnews.org

"There's enough data that we want to be looking at this, but we don't know yet for sure," says Ruth Carper, associate research professor of psychology at San Diego State University in California, who studies autism from early development through older adulthood.

Establishing proof of a link between autism and neurodegeneration could take decades, but in the meantime, the work could offer another benefit: understanding what autism looks like in the elderly and how best to support them.

"We are recognizing more and more people with autism in their 60s and 70s," says Sergio Starkstein, a neurologist and professor of psychiatry at the University of Western Australia in Perth, "and it's crucial to know how they are aging."

\section{Challenging diagnoses:}

Studies of older autistic adults are not easy to conduct. For one thing, autism is a relatively new diagnosis: Gruyna Sukhareva first characterized the condition in 1925; Leo Kanner described it in 1943. But the condition's diagnostic criteria have changed several times since.

People diagnosed 50 years ago are likely different from those diagnosed 5 years ago, Carper says - a 'cohort effect' that can make it difficult to extrapolate findings across generations. Also, given that timeline, many older adults with autism have never been diagnosed, she says. "There might be plenty of people who are 70 years old and have autism, but never got the label, so we can't find them."

Even when researchers can recruit older autistic adults, it's often difficult to diagnose them with a neurodegenerative condition. Many autistic people already have Parkinson's-like motor issues, such as a rigid gait or trouble coordinating movements. And there is overlap between autism traits and dementia in terms of cognitive deficits, particularly with executive-function skills such as planning and problem-solving, Vivanti says.

To date, there are no clinical guidelines on how to identify dementia in autistic adults using the cognitive tests designed for non-autistic adults, especially for those who are minimally verbal, he says.

Attempts to identify tangles, plaques and other signs of neurodegeneration in autistic people by way of brain imaging aren't straightforward either. The brains of autistic people can appear structurally different from those of non-autistic people from an early age, which makes interpreting a brain scan challenging, Starkstein says.

Starkstein became interested in the potential overlap between autism and neurodegenerative conditions after observing that many of the people he saw in his autism clinic had symptoms associated with Parkinson's disease. He conducted a follow-up study in 2015 and found that these 


\section{Spectrum | Autism Research News}

https://www.spectrumnews.org

traits affected 12 out of 37 of his autistic patients older than 39.

Joseph Piven, professor of psychiatry at the University of North Carolina at Chapel Hill, recruited another 19 autistic men in his own clinic for the same study and found that three had tremors and other motor symptoms related to Parkinson's.

Some of these motor problems are common side effects of antipsychotic medications, which many adults with autism take, Starkstein notes, but even among 20 people in the cohort who were not taking these drugs at the time of assessment, 4 had Parkinson's disease. "This proportion is far higher than what you would expect in a non-autistic population," Starkstein says.

Similarly high proportions of Parkinson's-like traits have surfaced among self-reports collected from 296 older autistic adults in the Netherlands and 209 in the United States. Nearly 20 percent of people in the Dutch cohort and more than 30 percent in the U.S. cohort described experiencing stiffness, tremors, slow movements and trouble with buttoning and dressing, among other motor challenges.

It's premature to conclude that autistic adults are more prone to Parkinson's disease than nonautistic people are based on these results, says senior investigator Hilde Geurts, professor of clinical neuropsychology at the University of Amsterdam. But she and her colleagues want to confirm or refute the findings by testing the motor skills of all study participants with an in-depth neurological assessment, she says.

\section{Converging results:}

Some researchers have turned to large health registries to garner evidence. In 2015, a study of thousands of medical records from healthcare organization Kaiser Permanente suggested that Parkinson's disease is about 30 times as common in adults with autism as in the general population. The study also found that autistic adults are about four times as likely to have dementia as controls are.

The dementia findings are in line with Vivanti's registry-based results: His team found that the prevalence of dementia is about 4 percent among adults with autism only, and more than 5 percent in those with autism and intellectual disability - much higher than the prevalence of dementia in people without autism or intellectual disability, which is less than 1 percent.

Piven cautions against relying solely on health records without assessing people in person. In his own work, he has found older people with autism diagnoses in their medical records who turned out to have frontotemporal dementia, a condition that leads to severe social difficulties, which clinicians had confused with the social difficulties associated with autism. But the convergence of 


\section{Spectrum | Autism Research News}

https://www.spectrumnews.org

findings across multiple studies of different kinds is not a huge surprise, he says.

Other neurodevelopmental conditions are associated with neurodegenerative disorders, Piven notes: People with 22q11.2 deletion syndrome, a genetic condition linked to autism, have an increased risk of early-onset Parkinson's disease, for example, and those with Down syndrome have high rates of dementia and Alzheimer's.

Why autistic people may have a higher risk of developing neurodegenerative diseases is unknown, although some researchers speculate that, as with other neurodevelopmental conditions, there is a genetic overlap. Chemical messengers, including serotonin and dopamine, are implicated in both autism and Parkinson's, and several genes involved in brain growth have cropped up in autism and dementia alike. What's more, compared with non-autistic people, autistic people have higher levels of beta-amyloid, the protein that forms plaques in the brains of people with Alzheimer's, according to analyses of blood samples and postmortem brains.

Any increased risk of dementia on the spectrum may also be related to lifestyle, Vivanti says. Engaging in intellectual, educational and social opportunities can protect against cognitive decline and dementia, research shows. "It's possible — but it is just speculation — that individuals with autism often experience barriers to accessing these opportunities," he says. In support of that idea, autistic teenagers show a loss of adaptive skills when they leave school, according to one 2020 study.

"Autistic people drop behind in terms of practical skills when they leave a structured environment that's not the same as degeneration, but it's a well-documented fact," says Catherine Lord, distinguished professor of psychiatry at the University of California, Los Angeles, and a senior investigator on the study.

For about 30 years, Lord has followed dozens of people with autism, taking baseline behavioral and neurobiological measurements starting when the participants reach their mid-thirties. Going forward, clinicians will need to track such cohorts for even longer, she says, and develop better tools to reliably diagnose motor or cognitive deteriorations across the entire spectrum — including in people who are minimally verbal or have severe intellectual disability.

That's the only way researchers will ever be able to establish a definitive link between autism and neurodegeneration, Lord says - "It's just crazy to have to wait 50 years."

Cite this article: https://doi.org/10.53053/FCYP3967 